\title{
Pilot study using virtual 4-D tracking electromagnetic navigation bronchoscopy in the diagnosis of pulmonary nodules: a single center prospective study
}

\author{
Tsukasa Ishiwata $^{1}$, Hideki Ujiie ${ }^{1}$, Alexander Gregor ${ }^{1}$, Terunaga Inage ${ }^{1}$, Yamato Motooka ${ }^{1}$, \\ Tomonari Kinoshita ${ }^{1}$, Masato Aragaki ${ }^{1}$, Zhenchian Chen ${ }^{1}$, Andrew Effat ${ }^{1}$, Nicholas Bernards ${ }^{1}$, \\ Kazuhiro Yasufuku ${ }^{1,2,3,4}$
}

${ }^{1}$ Division of Thoracic Surgery, Toronto General Hospital, University Health Network, Toronto, Ontario, Canada; ${ }^{2}$ Princess Margaret Cancer Centre, University Health Network/University of Toronto, Toronto, Ontario, Canada; ${ }^{3}$ TECHNA Institute for the Advancement of Technology for Health, University Health Network, Toronto, Ontario, Canada; ${ }^{4}$ Institute of Biomedical Engineering, University of Toronto, Toronto, Ontario, Canada Contributions: (I) Conception and design: All authors; (II) Administrative support: K Yasufuku; (III) Provision of study materials or patients: T Ishiwata, H Ujiie, K Yasufuku; (IV) Collection and assembly of data: T Ishiwata, H Ujiie; (V) Data analysis and interpretation: T Ishiwata, H Ujiie, A Gregor, T Inage, Y Motooka, T Kinoshita, M Aragaki, Z Chen, A Effat, N Bernards; (VI) Manuscript writing: All authors; (VII) Final approval of manuscript: All authors.

Correspondence to: Dr. Kazuhiro Yasufuku. Division of Thoracic Surgery, Toronto General Hospital, University of Toronto, 200 Elizabeth St, 9N-957, Toronto, Ontario M5G 2C4, Canada. Email: kazuhiro.yasufuku@uhn.ca.

Background: Electromagnetic navigation bronchoscopy (ENB) is a navigation technology intended to improve the diagnostic yield of pulmonary nodules. However, nodule displacement due to respiratory motion may compromise the accuracy of the navigation guidance. The Veran SPiNDrive ENB system employs respiratory-gating (4D-tracking) to compensate for this motion. The aim of the present study was to evaluate the diagnostic performance and safety of the Veran SPiNDrive system for biopsy of pulmonary nodules.

Methods: Adult patients with pulmonary nodules of $\geq 1 \mathrm{~cm}$ were enrolled at a single center. Both conventional bronchoscopy and 4D-tracking ENB were performed in one procedure session under general anesthesia, with the procedure order being randomly assigned. Radial probe endobronchial ultrasound and fluoroscopy were used in both groups. The diagnostic performance, safety, total procedure time, and total fluoroscopy time of the ENB phase were compared to the corresponding conventional bronchoscopy phase.

Results: The study was terminated due to poor accrual; a total of eleven patients were enrolled. The mean size of pulmonary nodules was $2.1 \mathrm{~cm}$. The sensitivity for malignancy was $67 \%(6 / 9)$ and $56 \%(5 / 9)$ with conventional bronchoscopy and with 4D-tracking ENB, respectively. Two cases developed minor bleeding after conventional bronchoscopy, while no complications were observed after 4D-tracking ENB. The mean procedure time was 16.1 and $21.7 \mathrm{~min}(\mathrm{P}=0.090)$, and the mean duration time for fluoroscopy use was 77 and $44 \sec (\mathrm{P}=0.056)$ for the conventional bronchoscopy and the 4D-tracking ENB phases, respectively.

Conclusions: The diagnostic performance of the Veran SPiNDrive 4D-tracking ENB did not exceed that of conventional bronchoscopy for pulmonary nodules. No complications were seen during 4D-tracking ENB. A study with a larger number of participants is required for further assessment.

Keywords: Electromagnetic navigation bronchoscopy; prospective study; 4D tracking; diagnostic performance

Submitted Jan 26, 2021. Accepted for publication Mar 25, 2021.

doi: $10.21037 /$ jtd-21-141

View this article at: http://dx.doi.org/10.21037/jtd-21-141

^ ORCID: 0000-0003-2369-2698. 


\section{Introduction}

Increasing use of low-dose computer tomography (CT) for lung cancer screening has improved the detection of solitary pulmonary lesions (1). The number of lung nodules detected incidentally is expected to increase in the future with broader lung cancer screening. Guidelines recommend the least invasive and safest diagnostic method possible, considering patients' probability of malignancy and risk of complications (2). If pulmonary nodules are strongly suspected to be malignant based on clinical and imaging investigations, surgical resection, such as videoassisted thoracoscopic surgery, is the recommended strategy satisfying the needs of both diagnosis and further treatment. When the probability of malignancy is moderate, further diagnostic testing such as CT-guided transthoracic needle aspiration (CT-TTNA) and/or flexible bronchoscopy is recommended $(3,4)$. CT-TTNA is undoubtedly an accurate modality for the diagnosis of pulmonary lesions, with more than $90 \%$ sensitivity $(5,6)$. However, the complication rate of CT-TTNA is high; pneumothorax occurs in $25-30 \%$ of procedures, with chest tube placement required in $4-15 \%$ of overall patients. In contrast, the bronchoscopic approach for pulmonary lesions has a safer profile than CT-TTNA $(7,8)$.

The prospective multicenter American College of Chest Physicians Quality Improvement Registry, Evaluation and Education (AQuIRE) registry revealed the persistent challenge for bronchoscopic diagnosis of pulmonary nodules; the sensitivity of conventional bronchoscopy for pulmonary nodules by transbronchial needle aspiration is $65 \%$ with fluoroscopic guidance, and $62 \%$ with radial probe endobronchial ultrasound (RP-EBUS) (9). RP-EBUS has become a critical tool for localization of pulmonary nodules (10). However, RP-EBUS still relies on accurate navigation to the target region. Conventionally, bronchoscopists view two-dimensional (2D) CT images and determine the path to the pulmonary nodule. However, one study demonstrated that the accuracy of path selection based on 2D-CT images was generally poor and largely depended on bronchoscopists' skills (11).

Electromagnetic navigation bronchoscopy (ENB) is a navigation technique that generates a virtual $3 \mathrm{D}$ reconstruction of the tracheobronchial tree from preprocedural CT data and superimposes the position of electromagnetic sensor-embedded instruments as they move within an electromagnetic field (12). The field generator is positioned outside the patient and is registered to the preprocedural images at the start of navigation bronchoscopy. A limitation of navigation bronchoscopy is the reliance on preoperative images, which do not account for differences in nodule position during different phases of ventilation. The Veran SPiNDrive navigation bronchoscopy system attempts to address this by employing both preoperative inhalation and exhalation CT scans for route generation as well as instrument tracking. Sensors on the patient's body allow for tracking of respiratory motion, and then update the instrument position accordingly (4D-tracking ENB, with the fourth dimension being time) (13). The Veran SPiNDrive system employs sampling devices with sensors embedded within, permitting continuous tracking during navigation and tissue acquisition.

The purpose of this prospective study was to assess the diagnostic performance of the Veran SPiNDrive 4D-tracking ENB system for pulmonary nodules and its safety. Unfortunately, this study was terminated before reaching our planned sample size due to slow accrual. The report here summarizes the diagnostic performance and the safety from the successfully enrolled patients.

We present the following article in accordance with the STARD reporting checklist (available at http://dx.doi. org/10.21037/jtd-21-141).

\section{Methods}

\section{Study plan and subjects}

This was a single-center prospective pilot study approved by the Institutional Review Board of the University Health Network (REB \#12-5056), and conducted at Toronto General Hospital (Toronto, Canada) between October 2013 and August 2017. Health Canada approved the use of the navigational bronchoscopy system for this study (ITA application \#195583). Written informed consent was obtained from all patients. This study was registered at the ClinicalTrials.gov registry (NCT01947530). The study was conducted in accordance with the Declaration of Helsinki (as revised in 2013). The target accrual was 20 patients over 2 years.

The inclusion criteria for this study were adult patients aged 18 and older with undiagnosed pulmonary nodules of $1 \mathrm{~cm}$ size or larger, and who were able to tolerate diagnostic bronchoscopy under general anesthesia. A pulmonary nodule was defined in this study as a lesion invisible through conventional bronchoscopy. The following patients 
were excluded: (I) patients with coagulopathy (defined as international normalized ratio $>2.0$ and/or prothrombin time $>2 \mathrm{x}$ normal), (II) pregnant patients, (III) patients with implantable devices susceptible to radiofrequency fields (e.g., implantable cardioverter-defibrillator), or (IV) morbidly obese patients. The morbid obesity exclusion was set by the manufacturer, to reduce the risk of sensor displacement between CT scan acquisition and bronchoscopy (see 'Study procedures').

\section{Study design}

Patients enrolled in the study underwent both conventional bronchoscopy and 4D-tracking ENB during the same procedure session. The order of the two procedures was assigned using a computer-based randomization system. The bronchoscopist performing conventional bronchoscopy was different from the bronchoscopist performing 4D-tracking ENB. Due to the technical training required for using the $4 \mathrm{D}$-tracking $\mathrm{ENB}$ system, the same bronchoscopist performed 4D-tracking ENB in all patients. All participating bronchoscopists were certified and wellexperienced thoracic surgeons or pulmonologists. Each bronchoscopist was blinded to the intraprocedural findings of the other. In addition, the conventional bronchoscopist was blinded to the route planning phase of the 4D-tracking ENB system.

\section{Study procedures}

The Veran SPiNDrive ${ }^{\mathrm{TM}}$ (SYS-1000, 2012, Veran Medical Technologies, Inc, St Louis, Missouri, USA) was used to perform 4D-tracking (Figure 1). All procedures were conducted in the guided therapeutics operating room at Toronto General Hospital (14), which includes an integrated CT scanner and fluoroscopy platform. Patients underwent preoperative chest CT scan with $0.8 \mathrm{~mm}$ slice thickness in both inspiratory and expiratory phases on the day of bronchoscopy with fiducial pads ( $\mathrm{vPad}^{\mathrm{TM}}$ ) placed on the anterior chest. Using the CT data and the position of the $\mathrm{vPad}$, route planning was conducted on the SPiNDrive system. The bronchoscopist assigned to perform conventional bronchoscopy was blinded to the process and results of the SPiNDrive plan, but had access to the preprocedural 2D-CT images for approach planning.

All bronchoscopic procedures were conducted under general anesthesia with neuromuscular blockade followed by either laryngeal mask airway placement or endotracheal intubation. Physiological monitoring was managed by a dedicated anesthesia team. While the first bronchoscopy procedure was performed (whether conventional bronchoscopy or 4D-tracking ENB), the second bronchoscopist was kept out of the room to blind them to any intraprocedural findings. A flexible bronchoscope with an outer diameter of $4.0 \mathrm{~mm}$ (BF-MP160F, Olympus, Japan) or $4.2 \mathrm{~mm}$ (BF-P190, Olympus, Japan) was used in both bronchoscopic procedures. The choice of bronchoscope was made at the start of the procedure; both conventional bronchoscopy and 4D-tracking ENB were performed with the same bronchoscope. RP-EBUS (UM-S20-17S, Olympus, Japan) and fluoroscopy (Artis Zeego system, Siemens, Germany) were used to confirm the target location before sampling in both conventional bronchoscopy and 4D-tracking ENB. For sampling specimens, transbronchial needle aspiration with a $21 \mathrm{G}$ needle (NA-401D-1321/NA-401D-1521, Olympus, Japan) was performed during conventional bronchoscopy, while Always-On Tip Tracked ${ }^{\mathrm{TM}}$ instruments (21 G needle, INS-0390; biopsy forceps, INS-0370; brush, INS-0350; Veran Medical Technologies, Inc, St Louis, Missouri, USA) were used during 4D-tracking ENB. At minimum, $21 \mathrm{G}$ needle aspiration was always used with $4 \mathrm{D}$-tracking ENB. Biopsy forceps and brushes were used at the bronchoscopist's discretion. The tip of each sampling device used with the SPiNDrive system has sensors embedded allowing continuous tracking within the electromagnetic field. A guide sheath was not used in these procedures. Rapid-on site evaluation cytology was not used in this study. Samples obtained with needles were processed in methanol-containing fixative (CytoLyt; Cytyc Corporation; Marlborough, Massachusetts, USA) for preparation of cell blocks. Brushing smears were prepared and fixed in $95 \%$ alcohol. Tissue specimens from forceps biopsies were placed in $10 \%$ formalin for histologic evaluation. All specimens were promptly delivered to the cytology laboratory after collection.

\section{Outcomes}

The primary outcome of this study was the sensitivity for malignancy with each technique. Final pathological diagnosis was confirmed via surgical resection or was confirmed as benign based on serial chest imaging. The secondary outcomes were safety, total procedure time, and total fluoroscopy time in each bronchoscopy procedure. Procedure time was measured from bronchoscope 
A

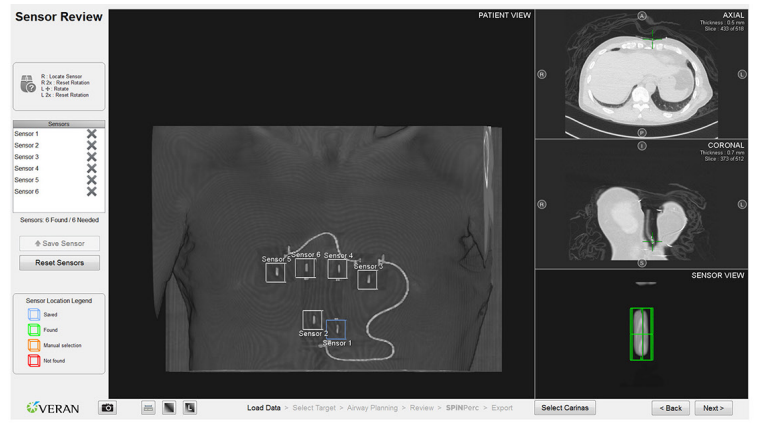

B

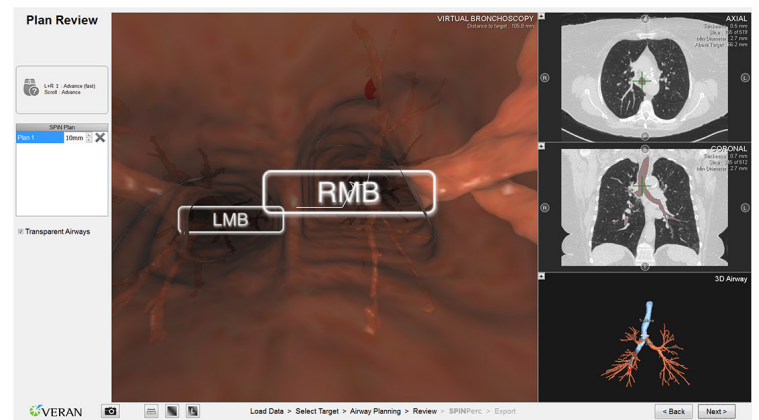

C

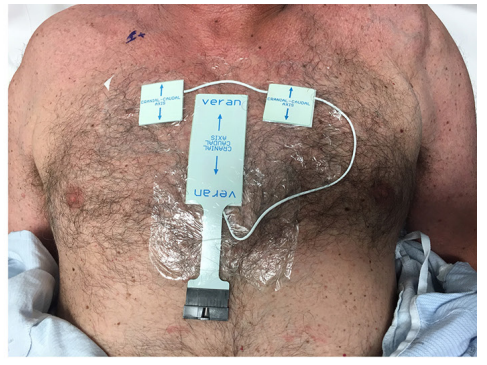

D

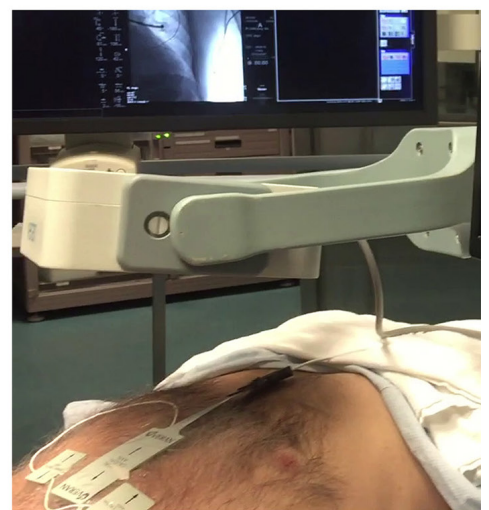

E

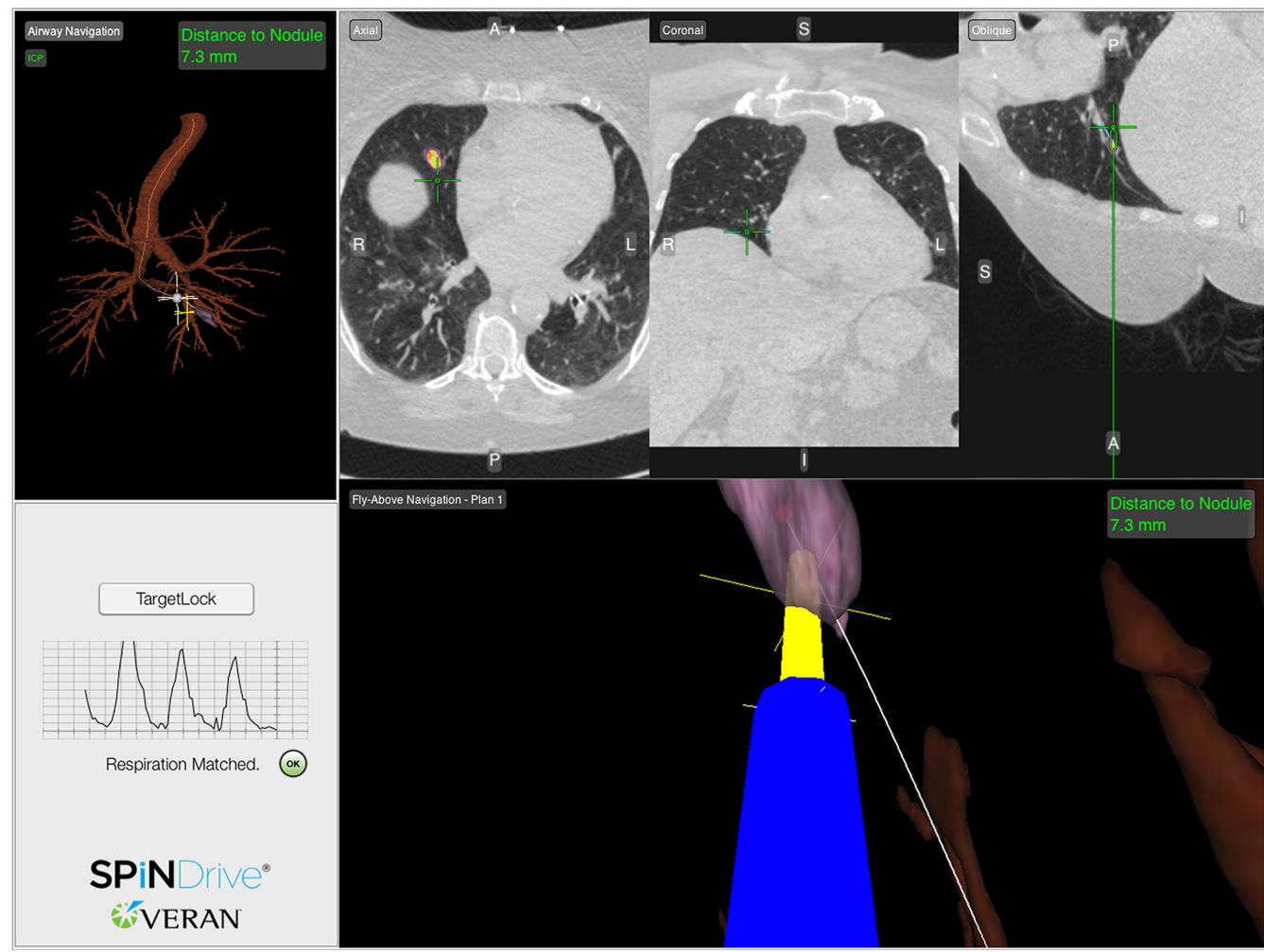

Figure 1 Planning and procedure images of 4D-tracking electromagnetic navigation bronchoscopy using the Veran SPiNDrive ${ }^{\mathrm{TM}}$ system. (A) In the planning phase, inspiratory and expiratory chest computed tomography images with chest fiducial pads (vPads ${ }^{\mathrm{TM}}$ ) are imported into the planning software. (B) Virtual fly-through images and a navigation route are generated. (C) Fiducial markers on the chest allows automatic registration of the preoperative images with the electromagnetic field, (D) the latter being produced by a generator placed above the patient's body during bronchoscopy. (E) The location of the sampling device relative to the preprocedural images and expected target position is shown in the virtual views, including estimated distance to target. 
Table 1 Patient characteristics and lesion data

\begin{tabular}{|c|c|}
\hline Patient characteristics and lesion variables & Value $(n=11)$ \\
\hline Age, median [range], y & 67 [57-90] \\
\hline \multicolumn{2}{|l|}{ Gender, n } \\
\hline Male & 7 \\
\hline Female & 4 \\
\hline Lesion size, mean (range), $\mathrm{cm}$ & $2.1(1.0-4.4)$ \\
\hline \multicolumn{2}{|l|}{ Lobe, $\mathrm{n}$} \\
\hline RUL & 5 \\
\hline RML & 1 \\
\hline RLL & 1 \\
\hline LUL & 4 \\
\hline LLL & 0 \\
\hline \multicolumn{2}{|l|}{ Location, $\mathrm{n}$} \\
\hline Central & 0 \\
\hline Intermediate & 4 \\
\hline Peripheral & 7 \\
\hline \multicolumn{2}{|l|}{ Bronchus sign, $\mathrm{n}$} \\
\hline Present & 9 \\
\hline Absent & 2 \\
\hline Distance to pleura, mean (range), $\mathrm{cm}$ & $1.9(0-5.3)$ \\
\hline \multicolumn{2}{|l|}{ Pathological diagnosis } \\
\hline Adenocarcinoma & 6 \\
\hline Squamous cell carcinoma & 2 \\
\hline Typical carcinoid & 1 \\
\hline Hamartoma & 1 \\
\hline Other benign disease & 1 \\
\hline
\end{tabular}

RUL, right upper lobe; RML, right middle lobe; RLL, right lower lobe; LUL, left upper lobe; LLL, left lower lobe.

insertion to bronchoscope removal. Adjustment of the electromagnetic field generator and registration were included in the procedure time for the ENB group.

\section{Statistical analysis}

Due to the slow accrual of participants, the study team took the decision to terminate the study before the target sample size could be reached. Statistical testing was still performed, but it was recognized the study was underpowered due to limited patient recruitment. Statistical analysis was performed using GraphPad Prism 8 (GraphPad Software, California, USA). Continuous variables are presented as means or medians with standard deviation (SD) or range. Categorical variables are presented as numbers and percentages. Two-tailed paired t-tests were conducted for comparing the continuous variables between conventional bronchoscopy and $4 \mathrm{D}$-tracking $\mathrm{ENB}$, with $\mathrm{P}<0.05$ considered statistically significant.

\section{Results}

A total of eleven patients were enrolled between October 2013 and August 2017. The original target was 20 patients over 2 years; slow accrual prompted the decision to close the study before reaching this sample size goal. The patient characteristics and lesion information are summarized in Table 1. The details of each patient and diagnostic outcomes are listed in Table 2. There were seven male and four female patients with a median age of 67 years (range, 57-90 years). The mean size of pulmonary nodules on CT was $2.1 \mathrm{~cm}$ (median, $2.0 \mathrm{~cm}$; range, $1.0-4.4 \mathrm{~cm}$ ). Nine pulmonary nodules $(82 \%)$ had a positive CT bronchus sign. Seven pulmonary nodules $(64 \%)$ were located in the peripheral third lung field. The mean pleural surface-to-pulmonary nodule distance was $1.9 \mathrm{~cm}$ (median, $1.9 \mathrm{~cm}$; range, 0-5.3 $\mathrm{cm}$ ). Representative CT images of the pulmonary nodules are shown in Figure 2. Seven cases underwent conventional bronchoscopy as the first procedure. Biopsy tools used for each case are shown in Table S1. Ten out of eleven patients underwent surgery for diagnosis and treatment. One patient (case \#4) was surveilled by imaging alone, with a total of three CT scans over two years; the nodule was clinically diagnosed as benign due to diminishing size.

As the primary outcome of this study, sensitivity for malignancy was $67 \%(6 / 9)$ and $56 \%(5 / 9)$ with conventional bronchoscopy and 4D-tracking ENB, respectively. In each patient, the RP-EBUS findings (concentric/eccentric/absent) in both groups were the same. Bronchoscopic navigation success, as shown by a concentric or eccentric view on RP-EBUS, was achieved in 6 out of 9 patients (67\%) with malignancy and 1 out of 2 patients $(50 \%)$ in benign nodules. There were two cases with minor bleeding (case \#3 and \#11) following conventional bronchoscopy, while no complications were observed following 4D-tracking ENB. In both cases, conventional bronchoscopy was the first procedure. No specific interventions were required to manage 


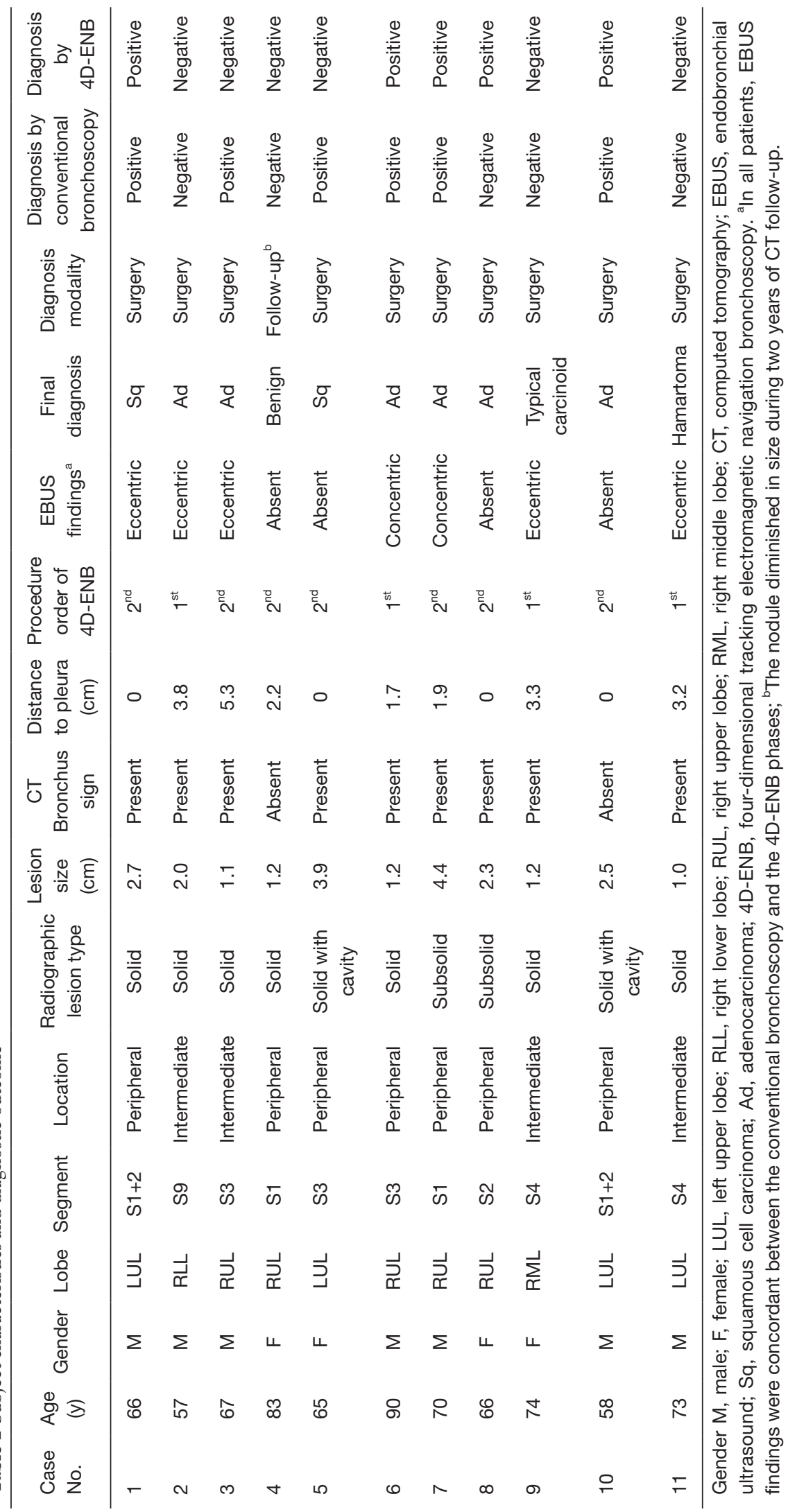


$\# 1$

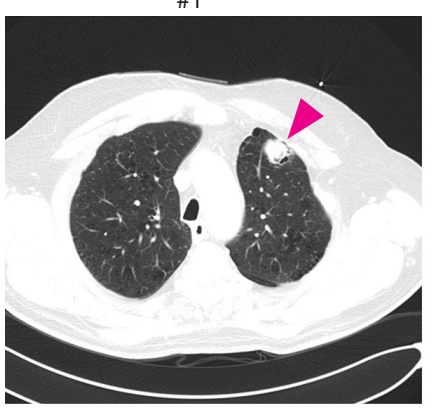

\#4

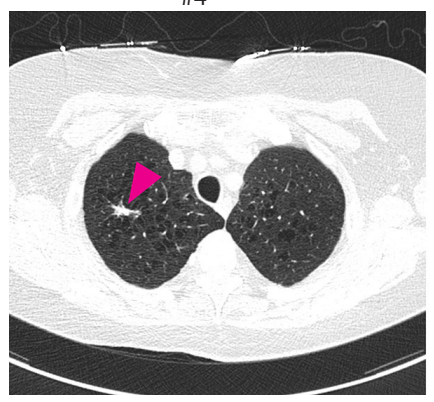

\#7

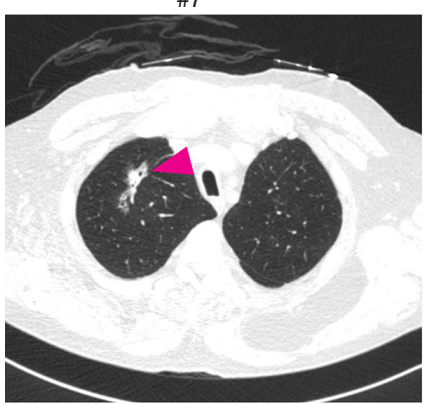

$\# 10$

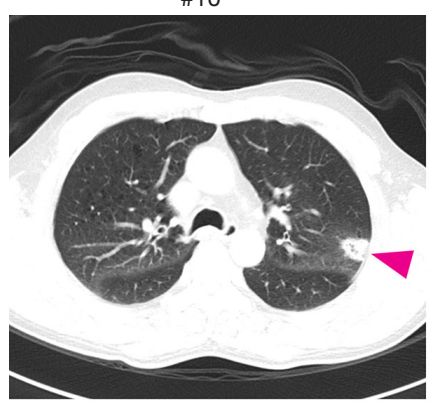

\#2

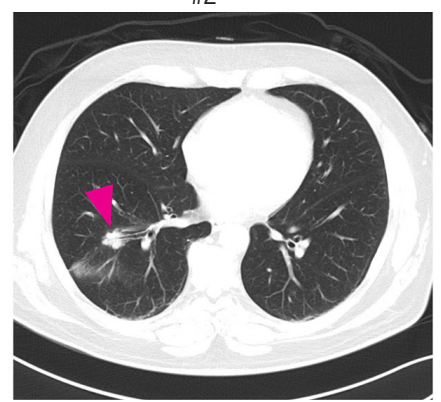

\#5

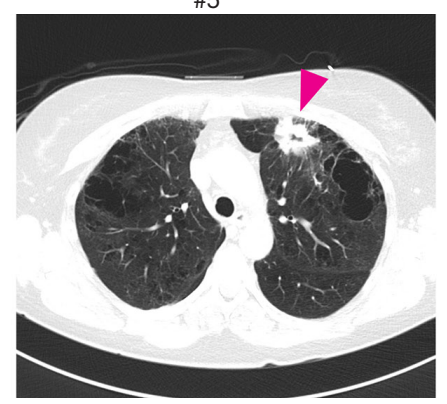

\#8

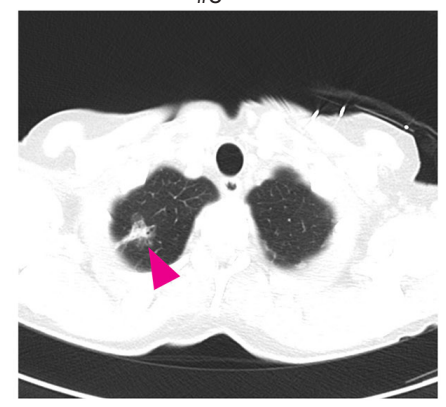

$\# 11$

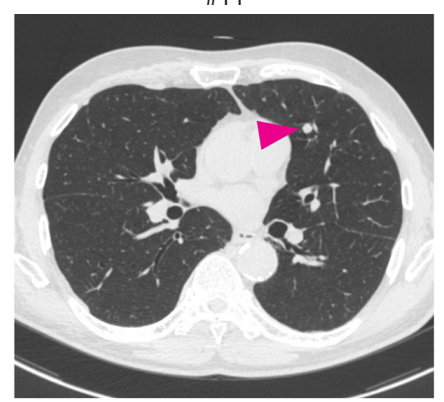

\#3

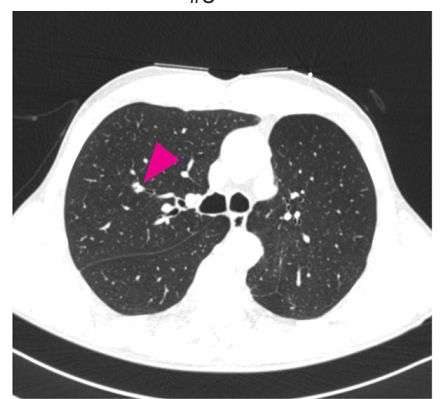

\#6

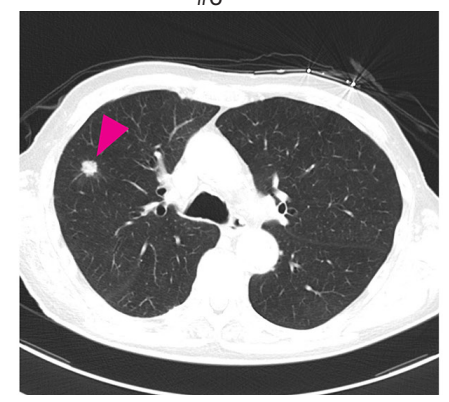

$\# 9$

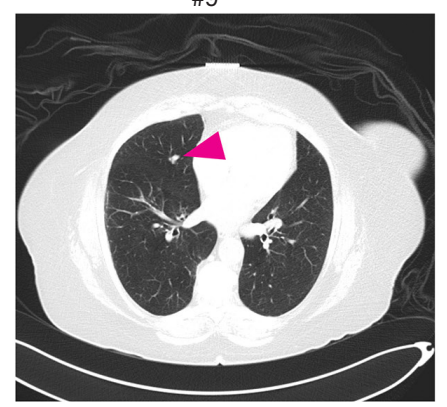

Figure 2 Computed tomographic axial images of the chest in all cases. Magenta arrow heads show locations of the target lesions.

the bleeding. The mean procedure time was $16.1 \mathrm{~min}$ (median, $16.0 \mathrm{~min}$; SD, $7.1 \mathrm{~min}$ ) and $21.7 \mathrm{~min}$ (median, $23.0 \mathrm{~min}$; $\mathrm{SD}, 8.7 \mathrm{~min})(\mathrm{P}=0.090)$ with conventional bronchoscopy and 4D-tracking ENB, respectively. The mean duration time for fluoroscopy use was $77 \mathrm{sec}(\mathrm{SD}$, $50 \mathrm{sec}$; mean dose-area product, $104.3 \mu \mathrm{Gy} \cdot \mathrm{m}^{2}$ ) and $44 \mathrm{sec}$ (SD, $25 \mathrm{sec}$; mean dose-area product, $69.1 \mu \mathrm{Gy} \cdot \mathrm{m}^{2}$ )
$(\mathrm{P}=0.056)$ with conventional bronchoscopy and 4D-tracking $\mathrm{ENB}$, respectively.

\section{Discussion}

This single center, prospective pilot study of 11 patients undergoing 4D-tracking ENB did not show an obviously 
higher sensitivity for malignancy than with conventional bronchoscopy when both groups underwent procedures using RP-EBUS and fluoroscopy to confirm the target location. No complications were observed with 4D-tracking ENB; however, the small sample size of this pilot study may have made it underpowered to detect complications.

This represents the first prospective study of Veran's 4D-tracking ENB for pulmonary nodules. The first ENB system approved by the FDA was superDimension ${ }^{\mathrm{TM}}$ (Medtronic, Minneapolis, Minnesota, USA). According to a recently published meta-analysis by Folch et al. (15), the superDimension ENB platform has a pooled sensitivity for malignancy of $78 \%$ (95\% CI, 73-83\%) in 38 studies. The 4D-tracking ENB's sensitivity for malignancy in our study was notably lower than this pooled analysis, at $56 \%$. One reason may have been that our study population included cases that were more difficult to approach for bronchoscopic diagnosis than other studies. Several authors have identified factors associated with improved ENB diagnostic yield, including larger size of pulmonary nodules $(16,17)$ and presence of a bronchus sign (16). In nine malignant nodules in this study, three were less than $1.5 \mathrm{~cm}$, and one nodule was in the peripheral third of the lung without a bronchus sign.

The navigation accuracy can be estimated by the ENB system-generated average fiducial target registration error (AFTRE). Lower AFTRE is associated with a higher diagnostic yield (18). A previous report of CT-guided sampling using a phantom demonstrated that the mean AFTRE of the Veran SPiNDrive ${ }^{\mathrm{TM}}$ system was $2.6 \mathrm{~mm}$ (13). A systematic-review by Gex et al. demonstrated that an overall pooled AFTRE from 11 studied using superDimension $^{\mathrm{TM}}$ was $5.1 \mathrm{~mm}$ (19). Theoretically, 4D-tracking should improve registration accuracy even further by accounting for respiratory motion, especially in lung nodules located in the lower lobes. A previous fundamental study about the dynamic movement of lung nodules during respiration by Chen et al. demonstrated that the average nodule displacement between inspiration and expiration was $12.2 \mathrm{~mm}, 10.6 \mathrm{~mm}, 25.3 \mathrm{~mm}$, and $23.8 \mathrm{~mm}$ in the right upper lobe, the left upper lobe, the right lower lobe, and the left lower lobe, respectively (20). Considering these findings, the effectiveness of respiratory gating in the 4D-tracking system might be most beneficial when targeting lower lobe pulmonary nodules. However, in our study, only one nodule was in the lower lobe, and as a result we may be underestimating the clinical utility of 4D-tracking ENB.
No complications were observed after 4D-tracking ENB in this study, and only two cases of minor bleeding were observed after conventional bronchoscopy. According to a meta-analysis on ENB (15), the rate of complications is generally low: $2.0 \%$ pneumothorax, $1.0 \%$ minor bleeding, and $0.8 \%$ major bleeding. ENB may be a safer technique than transthoracic biopsy; a meta-analysis including 32 studies found a pooled overall complication rates for transthoracic core biopsy and fine needle aspiration were $38.8 \%$ and $24.0 \%$, and the pooled pneumothorax rates were $25.3 \%$ and $18.0 \%$, respectively (21). However, the specific complication risk of ENB, especially pneumothorax, in the subgroup of patients with pulmonary nodules in the peripheral third lung field has not been well evaluated. A previous study by Mahajan et al. assessed the complication rates of performing ENB in patients with peripheral pulmonary nodules located at the fourth order bronchi or beyond; the overall pneumothorax rate was $10 \%(5 / 49)$, and 2 patients required chest tube placement, suggesting ENB in the peripheral third of the lung may be higher risk than pooled meta-analysis results may suggest (22). Therefore, fluoroscopy to prevent pneumothorax in such 'high-risk' pulmonary nodules is worth consideration. In our study, fluoroscopy was used in all cases, although several studies have demonstrated that there may be no significant difference in diagnostic yield between ENB with and without fluoroscopic assistance $(15,19)$. The fluoroscopy exposure time was shorter with 4D-tracking ENB than with conventional bronchoscopy. The real-time feedback and tracking during ENB may reduce the fluoroscopy exposure required for navigation to the target pulmonary nodule.

Our study also compared the procedure time between the two techniques. To the best of our knowledge, there have been no double-arm trials comparing the procedure time between ENB and conventional bronchoscopy. Our study did not show a significant difference in the procedure time between conventional bronchoscopy and 4D-tracking ENB, although some reports found that ENB tends to prolong procedure time $(19,23)$. Although procedure time has not been assessed in a meta-analysis to date, an interim analysis of a high-volume prospective study (NAVIGATE) using the superDimension ${ }^{\mathrm{TM}}$ ENB in 1215 subjects showed that the median total procedure time (bronchoscope insertionwithdrawal) was $52.0 \mathrm{~min}$ including $25.0 \mathrm{~min}$ of ENBspecific navigation and sampling time (24). By comparison, the median procedure time (bronchoscope insertionwithdrawal) using the Veran ENB system in our study was $23.0 \mathrm{~min}$. However, we cannot conclude that the Veran 
ENB system definitively reduces procedure time compared to the superDimension ${ }^{\mathrm{TM}} \mathrm{ENB}$, as clearance of secretions before sampling was omitted in seven cases as they had been performed during the initial conventional bronchoscopy as part of their randomization.

To improve the diagnostic yield of bronchoscopy, various novel technologies have been developed, such as robotic bronchoscopy for approaching peripheral pulmonary nodules with greater reach and stability $(25,26)$, and conebeam CT-guided bronchoscopy for accurate confirmation of target access (27). Previously published papers around these technologies show promising data, although more evidence is needed to conclude their usefulness. However, this does not mean that ENB is a technology of the past. As has been mentioned in reviews (12), a combination of factors is essential for bronchoscopic diagnosis of pulmonary nodules: (I) selecting the correct airway to reach the target (navigation), (II) approaching the target as close as possible (peripheral access), (III) confirming the location of the target before sampling (confirmation), and (IV) sampling the same place as confirmed prior (realtime feedback). As a navigational modality, robust evidence on ENB has accumulated regarding its safety $(15,24)$, diagnostic performance $(15,19)$, and cost-effectiveness $(28)$.

There are some limitations in this study. First, this was a pilot study with a limited number of participants. This reduces statistical power and thus it is difficult to confidently conclude any difference in sensitivity for malignancy, safety, and procedure time between conventional bronchoscopy and 4D-tracking ENB. A larger, ideally multicenter, study is key to accurately compare these metrics. Second, these 11 cases were our initial experience of using the 4D-tracking ENB system. Although the procedure time of ENB in our study did not show a relation with case number, it is possible our technique with the $4 \mathrm{D}$-tracking system was still sub-proficient. Third, even though the procedural order of conventional bronchoscopy and 4D-tracking ENB was randomly assigned, there might be a bias of the first procedure affecting the performance of the second procedure; e.g., erythema on the mucosa caused by the passage of a bronchoscope or sampling devices may have provided a clue for the navigation for the second procedure, or an enlarged shadow of the target on the fluoroscopic image caused by biopsy in the first procedure may have misled the navigation in the second procedure. This effect of procedure order was part of the justification for the randomized study design. Fourth, the sampling method was not consistent in both groups. As transbronchial needle aspiration using a $21 \mathrm{G}$ needle is a standard sampling method in our institution, transbronchial needle aspiration using a Veran kit was consistent at least in the 4D-tracking ENB group. However, additional forceps biopsies and brushing cytology were at the bronchoscopist's discretion in the 4D-ENB group, and the number of samplings was not standardized in this study. It is possible that this affected the results.

In this study with a limited number of participants, the 4D-tracking ENB did not show superior sensitivity for malignancy to conventional methods. This may be related to lack of statistical power. However, another possibility is that 4D-tracking ENB has most benefit in specific clinical scenarios that were not well-represented in our study. 4D-tracking ENB may have limited additive value when used in combination with other adjuncts, such as RP-EBUS, fluoroscopy, or extensive experience with conventional bronchoscopic biopsy. A further consideration is that nodule displacement during respiration varies most in the lower lobes, and it may be these patients who demonstrate the greatest value for 4D-tracking ENB. As evidence of the diagnostic performance of the superDimension ENB system has accumulated since when our study first opened, comparing Veran's ENB with superDimension in a larger follow-up study would be valuable for any future trial evaluating the benefits of 4D-tracking function. Additionally, a study assessing the 4D-tracking ENB performance under moderate sedation instead of general anesthesia may also be needed as coughing and/or body movements could interfere with navigation.

\section{Conclusions}

We assessed the diagnostic performance and the safety of a 4D-tracking ENB for undiagnosed pulmonary nodules in a prospective single center pilot study. The sensitivity of malignancy of the 4D-tracking ENB did not exceed that of conventional bronchoscopy. No complications were seen during ENB. A study with a larger number of participants is required for further assessment.

\section{Acknowledgments}

The authors are grateful to Sam Santiago for radiology technologic support, and Ms. Judy McConnell and Ms. Alexandria Grindlay for clinical research coordination.

Funding: This work was supported by the William Coco Chair in Surgical Innovation for Lung Cancer; and 
instrument and monetary support were provided by Veran Medical Technologies, Inc (St Louis, Missouri, USA). Alexander Gregor is a Hold'em for Life Oncology Clinician Scientist Trainee at the University of Toronto and holds a Frederick Banting and Charles Best Doctoral Canada Graduate Scholarship from the Canadian Institutes of Health Research (FRN 170883). Masato Aragaki holds a Grants-in-Aid for Scientific Research-KAKENHI from the Japan Society for the Promotion of Science.

\section{Footnote}

Reporting Checklist: The authors have completed the STARD reporting checklist. Available at http://dx.doi.org/10.21037/ jtd-21-141

Data Sharing Statement: Available at http://dx.doi. org/10.21037/jtd-21-141

Conflicts of Interest: All authors have completed the ICMJE uniform disclosure form (available at http://dx.doi. org/10.21037/jtd-21-141). KY reports other from William Coco Chair in Surgical Innovation for Lung Cancer, and other from Veran Medical Technologies, during the conduct of the study. MA reports grants from Japan Society for the Promotion of Science, during the conduct of the study. AG reports grants from the Canadian Institutes of Health Research, grants from the University of Toronto Temerty Faculty of Medicine/Hold'em for Life Charity Challenge, during the conduct of the study. The other authors have no conflicts of interest to declare.

Ethical Statement: The authors are accountable for all aspects of the work in ensuring that questions related to the accuracy or integrity of any part of the work are appropriately investigated and resolved. The study was conducted in accordance with the Declaration of Helsinki (as revised in 2013). This study was approved by Health Canada (ITA application \#195583) and the Institutional Review Board of the University Health Network (REB \#125056). Written informed consent was obtained from all patients.

Open Access Statement: This is an Open Access article distributed in accordance with the Creative Commons Attribution-NonCommercial-NoDerivs 4.0 International License (CC BY-NC-ND 4.0), which permits the noncommercial replication and distribution of the article with the strict proviso that no changes or edits are made and the original work is properly cited (including links to both the formal publication through the relevant DOI and the license). See: https://creativecommons.org/licenses/by-nc-nd/4.0/.

\section{References}

1. Church TR, Black WC, Aberle DR, et al. Results of initial low-dose computed tomographic screening for lung cancer. N Engl J Med 2013;368:1980-91.

2. Rivera MP, Mehta AC, Wahidi MM. Establishing the diagnosis of lung cancer: Diagnosis and management of lung cancer, 3rd ed: American College of Chest Physicians evidence-based clinical practice guidelines. Chest 2013;143:e142S-e165S.

3. Zhan P, Xie H, Xu C, et al. Management strategy of solitary pulmonary nodules. J Thorac Dis 2013;5:824-9.

4. Elia S, Loprete S, De Stefano A, et al. Does aggressive management of solitary pulmonary nodules pay off? Breathe (Sheff) 2019;15:15-23.

5. DiBardino DM, Yarmus LB, Semaan RW. Transthoracic needle biopsy of the lung. J Thorac Dis 2015;7:S304-16.

6. Zhang HF, Zeng XT, Xing F, et al. The diagnostic accuracy of CT-guided percutaneous core needle biopsy and fine needle aspiration in pulmonary lesions: a metaanalysis. Clin Radiol 2016;71:e1-10.

7. Asano F, Aoe M, Ohsaki Y, et al. Deaths and complications associated with respiratory endoscopy: a survey by the Japan Society for Respiratory Endoscopy in 2010. Respirology 2012;17:478-85.

8. Zhan P, Zhu QQ, Miu YY, et al. Comparison between endobronchial ultrasound-guided transbronchial biopsy and CT-guided transthoracic lung biopsy for the diagnosis of peripheral lung cancer: a systematic review and metaanalysis. Transl Lung Cancer Res 2017;6:23-34.

9. Ost DE, Ernst A, Lei X, et al. Diagnostic Yield and Complications of Bronchoscopy for Peripheral Lung Lesions. Results of the AQuIRE Registry. Am J Respir Crit Care Med 2016;193:68-77.

10. Sainz Zuñiga PV, Vakil E, Molina S, et al. Sensitivity of Radial Endobronchial Ultrasound-Guided Bronchoscopy for Lung Cancer in Patients With Peripheral Pulmonary Lesions: An Updated Metaanalysis. Chest 2020;157:994-1011.

11. Dolina MY, Cornish DC, Merritt SA, et al. Interbronchoscopist variability in endobronchial path selection: a simulation study. Chest 2008;133:897-905.

12. Ishiwata T, Gregor A, Inage T, et al. Advances 
in interventional diagnostic bronchoscopy for peripheral pulmonary lesions. Expert Rev Respir Med 2019;13:885-97.

13. Appelbaum L, Sosna J, Nissenbaum Y, et al. Electromagnetic navigation system for CT-guided biopsy of small lesions. AJR Am J Roentgenol 2011;196:1194-200.

14. Ujiie H, Effat A, Yasufuku K. Image-guided thoracic surgery in the hybrid operation room. J Vis Surg 2017;3:148.

15. Folch EE, Labarca G, Ospina-Delgado D, et al. Sensitivity and Safety of Electromagnetic Navigation Bronchoscopy for Lung Cancer Diagnosis: Systematic Review and Metaanalysis. Chest 2020;158:1753-69.

16. Seijo LM, de Torres JP, Lozano MD, et al. Diagnostic yield of electromagnetic navigation bronchoscopy is highly dependent on the presence of a Bronchus sign on CT imaging: results from a prospective study. Chest 2010;138:1316-21.

17. Bowling MR, Kohan MW, Walker P, et al. The effect of general anesthesia versus intravenous sedation on diagnostic yield and success in electromagnetic navigation bronchoscopy. J Bronchology Interv Pulmonol 2015;22:5-13.

18. Makris D, Scherpereel A, Leroy S, et al. Electromagnetic navigation diagnostic bronchoscopy for small peripheral lung lesions. Eur Respir J 2007;29:1187-92.

19. Gex G, Pralong JA, Combescure C, et al. Diagnostic yield and safety of electromagnetic navigation bronchoscopy for lung nodules: a systematic review and meta-analysis. Respiration 2014;87:165-76.

20. Chen A, Pastis N, Furukawa B, et al. The effect of respiratory motion on pulmonary nodule location during electromagnetic navigation bronchoscopy. Chest 2015;147:1275-81.

Cite this article as: Ishiwata $\mathrm{T}$, Ujiie $\mathrm{H}$, Gregor A, Inage T, Motooka Y, Kinoshita T, Aragaki M, Chen Z, Effat A, Bernards N, Yasufuku K. Pilot study using virtual 4-D tracking electromagnetic navigation bronchoscopy in the diagnosis of pulmonary nodules: a single center prospective study. J Thorac Dis 2021;13(5):2885-2895. doi: 10.21037/jtd-21-141
21. Heerink WJ, de Bock GH, de Jonge GJ, et al. Complication rates of CT-guided transthoracic lung biopsy: meta-analysis. Eur Radiol 2017;27:138-48.

22. Mahajan AK, Patel S, Hogarth DK, et al. Electromagnetic navigational bronchoscopy: an effective and safe approach to diagnose peripheral lung lesions unreachable by conventional bronchoscopy in high-risk patients. J Bronchology Interv Pulmonol 2011;18:133-7.

23. Hautmann H, Schneider A, Pinkau T, et al. Electromagnetic catheter navigation during bronchoscopy: validation of a novel method by conventional fluoroscopy. Chest 2005;128:382-7.

24. Folch EE, Pritchett MA, Nead MA, et al. Electromagnetic Navigation Bronchoscopy for Peripheral Pulmonary Lesions: One-Year Results of the Prospective, Multicenter NAVIGATE Study. J Thorac Oncol 2019;14:445-58.

25. Chen AC, Pastis NJ, Jr., Mahajan AK, et al. Robotic Bronchoscopy for Peripheral Pulmonary Lesions: A Multicenter Pilot and Feasibility Study (BENEFIT). Chest 2021;159:845-52.

26. Yarmus L, Akulian J, Wahidi M, et al. A Prospective Randomized Comparative Study of Three Guided Bronchoscopic Approaches for Investigating Pulmonary Nodules: The PRECISION-1 Study. Chest 2020;157:694-701.

27. Casal RF, Sarkiss M, Jones AK, et al. Cone beam computed tomography-guided thin/ultrathin bronchoscopy for diagnosis of peripheral lung nodules: a prospective pilot study. J Thorac Dis 2018;10:6950-9.

28. Rickets W, Lau KKW, Pollit V, et al. Exploratory costeffectiveness model of electromagnetic navigation bronchoscopy (ENB) compared with CT-guided biopsy (TTNA) for diagnosis of malignant indeterminate peripheral pulmonary nodules. BMJ Open Respir Res 2020;7:e000595. 
Supplementary

Table S1 Types of sampling tools and the number of sampling passes used in each case.

\begin{tabular}{|c|c|c|}
\hline Case No. & Conventional bronchoscopy & 4D-ENB \\
\hline 1 & TBNA $\times 4$ & TBNA $\times 3$ \\
\hline 2 & TBNA $\times 3$ & TBNA $\times 5$ \\
\hline 3 & TBNA $\times 3$ & TBNA x2, Brush x1 \\
\hline 4 & TBNA x3 & TBNA x1, TBB x2, Brush x1 \\
\hline 5 & TBNA $\times 3$ & TBNA x3, Brush x1 \\
\hline 6 & TBNA $\times 2$ & TBNA $\times 2$ \\
\hline 7 & TBNA x3 & TBNA x1, Brush x1 \\
\hline 8 & TBNA x3 & TBNA x3, TBB x2, Brush x1 \\
\hline 9 & TBNA $\times 3$ & TBNA x3, TBB x4 \\
\hline 10 & TBNA x2 & TBNA x3, TBB x2, Brush x1 \\
\hline 11 & TBNA $\times 3$ & TBNA $\times 3$ \\
\hline
\end{tabular}

4D-ENB, four-dimensional electromagnetic navigation bronchoscopy; TBNA, transbronchial needle aspiration; TBB, transbronchial biopsy. 